\title{
Empirical Analysis of Duality Spaces Connecting Distinct Optical form Transforms
}

\author{
Padmaja G, Gulhane A
}

\begin{abstract}
This paper presents a novel technique of construction a precise functional frame in presence of the new proposed constraints during the planning straightforward extension of excessive considerable dimensional generalizations using a empirical relationship of two absolutely distinct transforms having diverse kernels transform for the Laplace Stieltjes spaces consisting of analytical signals from two dimensions at any point heavily affecting the successful development for the view of the Gelfand Shilov techniques a subspace of a Schwartz space simple objective function along with their duals implies continuity having functional analyst approach under many classical conventional transforms arise naturally as Laplace Stielties transform of certain distributions extensively used in many applications like magnetic field theory follows from the belongings of strong continuity at origin lean heavily in constructing multidimensional

$S$ type spaces based on the testing function spaces upto some desired order for infinitely differentiable functions $\varphi(t, x)$ with Gelfand Shilov concept under one umbrella.
\end{abstract}

Keywords: Laplace Stieltjes Transform, Continuous Linear Functional, Duality spaces, Multidimensional Sense, Gelfand Shilov Spaces.

\section{INTRODUCTION}

In order to develop sufficient well established valuable techniques having origin in well known work by Schwartz in the form of theory of distributional integral transforms also the work by Oliver Heaviside 1890 useful in further testing and comparing the capabilities of the numerious modern methods mainly for Dirac delta distributions suitably applied in modeling electronics as $\delta(t)$ equals to zero for nonzero functions and $\infty$ for $t$ equals to zero as increasing development of magnetic imaging stressed back guanine interest to solve partial differential equations involving distributional conditions like equation of propagation of heat in cylindrical coordinates imposing generalized boundary conditions especially in magnetic field theory by optimizing efforts the linear parts of equations the motivational work for distributional generalized Laplace-Stieltjes transform large impact configuration involving both integral and differentiation concepts under a single domain.

The special attention on image simulations of all stages the objects need focus on exposition due to wide spread

Revised Manuscript Received on June 12, 2020.

* Correspondence Author

Padmaja G*, Assistant Professor, Government College of Engineering, Amravati (Maharashtra), India.

Gulhane A, Research Assistant, University of Illinois at Urbana-Champaign, U.S.

(c) The Authors. Published by Blue Eyes Intelligence Engineering and Sciences Publication (BEIESP). This is an open access article under the CC BY-NC-ND license (http://creativecommons.org/licenses/by-nc-nd/4.0/) various properties \& applicability especially emerging from various scientific real life situations engineering fields indispensable many dimensions for achieving mostly where the exponential decay of clearly marked out continuous non-continuous determined functions $\varphi(t, x)=\varphi\left(t_{1},--, t_{m}, x\right)$ many dimensions for

achieving mostly where the exponential decay of clearly marked out continuous non-continuous determined functions $\varphi(t, x)=\varphi\left(t_{1},--, t_{m}, x\right)$ intensively studied with projective descriptions of a general class of Gelfand Shilov spaces of extension nature proposing new sets of test functions having the approach to solve various types of different order different degree ordinary differential equations partial differential equations upto some desired order over some particular sphere for decrease of respective polynomials in the variables $t_{1},--, t_{m}, x$ at infinity.

\section{MULTIDIMENSIONAL SPACES}

The recent achievements create the main interest historically with generalized functions also known as distributions the systematic theory of distributional integral classes relate this matter the asymptotic expansion for the derivative of dependent variable $\varphi(t, x)=\varphi\left(t_{1},--, t_{m}, x\right)$ respect to $t$ denoted by $\partial t_{1}{ }^{l}---\partial t_{m}{ }^{l}$ extend the aspect the real world problems not only makes it more complex but also characterized by increase potential equiparallel topology of the multinormed space $S_{\alpha, a}=S_{\alpha_{1},---, \alpha_{m}, a}=S_{\alpha_{1,---, \alpha_{m}, a}}\left(R^{d}\right)$ from above section for $t_{i}=t_{1},---, t_{m}$ the space increased number of decision variables and constraints generated by the countable multiform the Laplace Stieltjes transformation with distinct $m+1$ real(complex) variables inequality satisfied for the relation multidimensional scalable test functions $\gamma_{a, k, l, q} \varphi$ space is equal to

$$
\begin{gathered}
\sup _{\substack{0<t<\infty \\
0<x<\infty}}\left|e^{a\left(t_{1},--, t_{m}\right)}(1+x)^{k} D_{\left(t_{1},--, t_{m}\right)}^{l}\left(x D_{x}\right)^{q} \varphi\left(t_{i}, x\right)\right| \\
\leq C_{l} A_{1}^{a}---A_{m}{ }^{a} a^{a \alpha_{1}}---a^{a \alpha_{m}}
\end{gathered}
$$

Published By:

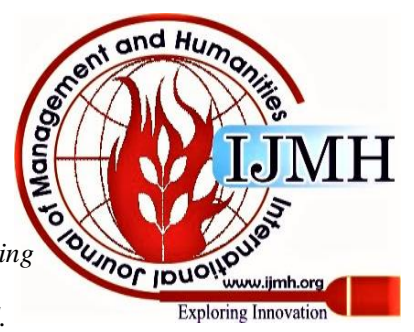




\section{Empirical Analysis of Duality Spaces Connecting Distinct Optical Form Transforms}

where growth in the fields are continuously proposing new improved algorithm to handle more general classes the constants $A_{1}---A_{m}$ casually depend respectively on the almost everywhere differentiable independent variables $t_{1},--, t_{m}$ and $C_{l}$ depend on the everywhere differentiable continuous function whose derivatives with respect to independent variables exists at all points on its domain explore the properties of testing function $\varphi\left(t_{i}, x\right)$ and $a \in R$. We get

$$
a^{a \alpha_{1}}=---=a^{a \alpha_{m}}=1 \text { for } a=0 \text {. }
$$

The idea leads to the concept of systematic study of magnetic domain structure presented as an application of the geometric objects and spatial relations unaffected preserved under the continuous change of shape size distortion of the multinormed space generated by the countable multiform $\left\{\gamma_{a, k, l, q}\right\}_{l, q=0}^{\infty}$

With these various arrangements of the elements network commonly used topologies $S_{\alpha, a}$ is a countably multiform complete normed real (or complex) strongest admissible possible one determined with an energy filtered continuous magnetic induction mapping technique implemented on $S_{\alpha, a_{v}}$ to $S_{\alpha, a}$ for every choice of $v>0$.

$$
\begin{aligned}
& \gamma_{a, k, l, q} \varphi= \\
& \sup _{\substack{0<t<\infty \\
0<x<\infty}}\left|e^{a\left(t_{1},---, t_{m}\right)}(1+x)^{k} D_{\left(t_{1},--, t_{m}\right)}^{l}\left(x D_{x}\right)^{q} \varphi\left(t_{i}, x\right)\right| \\
& \quad \leq C_{l} A_{1}^{a}---A_{m}{ }^{a} a^{a \alpha_{1}}---a^{a \alpha_{m}}
\end{aligned}
$$

according to situations the constants $C_{l}, A_{1},---, A_{m}$ where the behavior on functions $\varphi\left(t_{i}, x\right)$ as $m+1$ independent variables increases or decreases varying without bound approaches to $\infty$ eventhough it's impossible to see or feel without the right tool for suitable every choice of $v>0$ mentioned $S_{\alpha}$ is a countably multiform complete normed real (or complex) strongest possible one topology with $a_{v}$ continuous $m+1$ induction maps $S_{\alpha, a_{v}}$ to $S_{\alpha, a}$ for $\alpha=\alpha_{1},---, \alpha_{m}$.

The constraints studied having crucible role in mathematical analysis, mathematical physics and engineering of generalized functions are mainly converted on the growth of the partial derivatives instead the decrease of the function $\varphi(t, x)=\varphi\left(t_{1},---, t_{m}, x\right)$ at infinity respective to the polynomials in $t_{1},--, t_{m}, x$ for diversion study of exponential constructed space $S^{\beta, l}=S^{\beta_{1},---, \beta_{m}, l}$ with property

$\gamma_{a, k, l, q} \varphi=$

$$
\begin{array}{r}
\sup _{\substack{0<t<\infty \\
0<x<\infty}}\left|e^{a\left(t_{1},---, t_{m}\right)}(1+x)^{k} D_{\left(t_{1},--, t_{m}\right)}^{l}\left(x D_{x}\right)^{q} \varphi\left(t_{i}, x\right)\right| \\
\leq C_{a} B_{1}^{l}---B_{m}{ }^{l} l^{l \beta_{1}}---l^{l \beta_{m}}<\infty
\end{array}
$$

where the constants $B_{1},---B_{m}, C_{a}$ depend on $m+1$ independent variables continuous differentiable function $\varphi$ satisfying convergent continuous induction map from $S^{\beta_{\tau}, l}$ to $S^{\beta_{\tau}, l}$ every choice of $\tau>0 \quad \beta_{\tau}$ for mentioned $S^{\beta, l}$ a countably multiform complete normed real (or complex) strongest possible one topology as a application of differentiable functions whose derivatives do or donot exist in the classical sense for the space having constraints mainly on the growth of the involved partial derivatives as $l$ approaches to infinity for $\beta>0$ as the origin. The extensively used contribution for general engineering technical model for current magnetism the development of the necessary facts which carries great potential to impact areas such as data storage sensing biomagnetism hence the construction \& extension related to the generalized functions theory by Schwartz will be applied to the formal presentation of the proposed test function Laplace Stieljes transform determined and executed to preserve certain properties for theory of generalized distributional transform based on the application of natural transforms the test function space $S$ consisting of all infinitely differentiable function $\varphi(t, x)$ defined for all positive values of $t_{1},---, t_{m}, x$ having continuous derivative over some domain $C^{\infty}\left(R^{d_{i}}\right)$ satisfying

$$
\begin{gathered}
\gamma_{a, k, l, q} \varphi= \\
\sup _{\substack{0<t<\infty \\
0<x<\infty}}\left|e^{a\left(t_{1},--, t_{m}\right)}(1+x)^{k} D_{\left(t_{1},--, t_{m}\right)}^{l}\left(x D_{x}\right)^{q} \varphi\left(t_{i}, x\right)\right| \\
\leq C_{i} A_{1}^{a}---A_{m}{ }^{a} B_{1}^{l}---B_{m}{ }^{l} \\
a^{a \alpha_{1}}---a^{a \alpha_{m}} l^{l \beta_{1}}---l^{l \beta_{m}}<\infty
\end{gathered}
$$

Let there be given $\alpha_{i}, \beta_{i}>0, C_{i}, A_{i}, B_{i} \in R$ fixed such that $C_{i}=\left(C_{l}, C_{a}\right)$ with $\varphi(t, x)$ function defined for all positive values of $t_{1},---, t_{m}, x$ having continuous derivative over some domain $C^{\infty}\left(R^{d_{i}}\right)$. Gelfand Shilov type space connected to study the local regularity properties of the nanostructure making the determination highly valuable analyzing functions relative to kernel of Laplace transform $S_{\alpha_{i}, A_{i}}^{\beta_{i}, B_{i}}=S_{\alpha_{i}, A_{i}}^{\beta_{i}, B_{i}}\left(R^{d_{i}}\right)$ is defined by

$$
\begin{gathered}
S_{\alpha_{i}, A_{i}}^{\beta_{i}, B_{i}}=\left\{\varphi \in C^{\infty}\left(R^{d_{i}}\right) / \exists C_{i}>0,\right. \\
\sup _{\substack{0<t<\infty \\
0<x<\infty}}\left|e^{a\left(t_{1},--, t_{m}\right)}(1+x)^{k} D_{\left(t_{1},--, t_{m}\right)}^{l}\left(x D_{x}\right)^{q} \varphi\left(t_{i}, x\right)\right| \\
\leq C_{i} A_{1}^{a}---A_{m}{ }^{a} B_{1}{ }^{l}---B_{m}{ }^{l} \\
\left.a^{a \alpha_{1}}---a^{a \alpha_{m}} l^{l \beta_{1}}---l^{l \beta_{m}}\right\}
\end{gathered}
$$

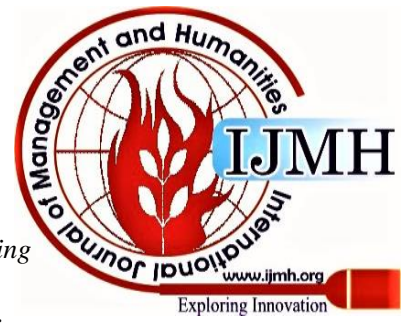


obviously the spaces $S_{\alpha_{i}, a}, S^{\beta_{i}, l}$ satisfying the user's requirements of open \& close management and visual query in theoretical practical technical forward models a novel scheme study are subspaces of the above testing function space for all non negative numbers $\alpha_{i}, \beta_{i}$ for $0<t_{i}<\infty, 0<x<\infty$ where the constants $C_{i}, A_{i}, B_{i}$ depend on the everywhere differential testing function $\varphi$. From a topological point of view the spaces $S_{\alpha_{i}}^{\beta_{i}}$ and $\sum_{\alpha_{i}}^{\beta_{i}}$ are given by the union and intersection for $A_{i}, B_{i} \geq 0$ of $S_{\alpha_{i}, A_{i}}^{\beta_{i}, B_{i}}$ respectively with their topologies having special paid attention on the inductive and projective limits:

$$
S_{\alpha_{i}}^{\beta_{i}}=\text { ind } \lim _{A_{i}, B_{i}>0} S_{\alpha_{i}, A_{i}}^{\beta_{i}, B_{i}}, \sum_{\alpha_{i}}^{\beta_{i}}=\text { proj } \lim _{A_{i}, B_{i}>0} S_{\alpha_{i}, A_{i}}^{\beta_{i}, B_{i}}
$$

$S_{\alpha_{i}}^{\beta_{i}}$ and $\sum_{\alpha_{i}}^{\beta_{i}}$ are nontrivial iff $\alpha_{i}+\beta_{i} \geq 0$ and $\alpha_{i} \beta_{i}>0$ the union and intersection for each and every constants related to $A_{i}, B_{i} \geq 0$ of $S_{\alpha_{i}}^{\beta_{i}, B_{i}}$.

The powerful approach presented here is widely applicable to a broad range of magnetic nanostructures evidently the space $S_{\alpha_{i}}^{\beta_{i}}$ of all non negative numbers $\alpha, \beta$ is contained in the intersection of the spaces $S_{\alpha_{i}, a}, S^{\beta_{i}, l}$ whereas space as a union of countably normed spaces were able to define sequential convergence in all metioned spaces such that these spaces became sequentially complete.

The Gelfand Shilov type distributional spaces $\left(S_{\alpha_{i}}^{\beta_{i}}\right)^{\prime}$ and $\left(\sum_{\alpha_{i}}^{\beta_{i}}\right)^{\prime}$ ' are given by the intersection and union for $A, B \geq 0$ of $\left(S_{\alpha_{i}, A_{i}}^{\beta_{i}, B_{i}}\right)^{\prime}$ and its topological sence is given by the projective and inductive limits:

$$
\left(S_{\alpha_{i}}^{\beta_{i}}\right)^{\prime}=\bigcap_{A_{1}, B_{1}>0}\left(S_{\alpha_{i}, A_{i}}^{\beta_{i}, B_{i}}\right)^{\prime} \text { ' and }\left(\sum_{\alpha_{i}}^{\beta_{i}}\right)^{\prime}=\bigcup_{A_{1}, B_{1}>0}\left(S_{\alpha_{i}, A_{i}}^{\beta_{i}, B_{i}}\right) '
$$

Here $\left(S_{\alpha_{i}}^{\beta_{i}}\right)^{\prime}$ is the dual of $S_{\alpha_{i}}^{\beta_{i}}$ and $\left(\sum_{\alpha_{i}}^{\beta_{i}}\right)$ ' is the dual of $\sum_{\alpha_{i}}^{\beta_{i}}$.

\section{EXPANDED HYBRIDIZATION}

Now as a special case a thorough understanding of the connectional relation between two different structures apply the distributional Stieltjes transformation over above generalized version of the classical suitable kernel exponential sense as well as polynomial approach that permits direct measurements of magnetic ordering at a suitable scale_relative to Gelfand Shilov type spaces $S_{\alpha_{j}, A_{j}}^{\beta_{j}, B_{j}}=S_{\alpha_{j}, A_{j}}^{\beta_{j}, B_{j}}\left(R^{d_{j}}\right)$ for convenience under proper coordination of the variables and parameters in a unified manner $C_{j}=\left(C_{q}, C_{k}\right)$ where the constants $C_{j}, A_{j}, B_{j}$ depend on the everywhere differential testing function $\varphi$ by

$$
S_{\alpha_{j}, A_{j}}^{\beta_{j}, B_{j}}=\left\{\varphi \in C^{\infty}\left(R^{d_{j}}\right) / \exists C_{j}>0,\right.
$$

$$
\begin{gathered}
\sup _{\substack{0<t<\infty \\
0<x<\infty}}\left|e^{a\left(t_{1},--, t_{m}\right)}(1+x)^{k} D_{\left(t_{1},--, t_{m}\right)}^{l}\left(x D_{x}\right)^{q} \varphi\left(t_{i}, x\right)\right| \\
\left.\leq C_{j} A^{k} B^{q} k^{k \alpha_{j}} q^{q \beta_{j}}\right\}
\end{gathered}
$$

The terminology discussed in spaces $S_{\alpha_{j}}^{\beta_{j}}$ and $\sum_{\alpha_{j}}^{\beta_{j}}$ are generally understood to mean the union and intersection on largely based unique constants $A_{j}, B_{j} \geq 0$ of $S_{\alpha_{j}, A_{j}}^{\beta_{j}, B_{j}}$ takes a new look at real field applications indicating the time frame in many continuous non-continuous processes with its appreciable topology given by the appropriate inductive and projective limits significantly

$$
\begin{aligned}
S_{\alpha_{j}}^{\beta_{j}} & =\text { ind } \lim _{A_{2}, B_{2}>0} S_{\alpha_{j}, A_{j}}^{\beta_{j}, B_{j}} \\
\sum_{\alpha_{j}}^{\beta_{j}} & =\text { proj } \lim _{A_{2}, B_{2}>0} S_{\alpha_{j}, A_{j}}^{\beta_{j}, B_{j}}
\end{aligned}
$$

Our substantial findings $S_{\alpha_{j}}^{\beta_{j}}$ and $\sum_{\alpha_{j}}^{\beta_{j}}$ are nontrivial iff the significant union intersection compare well with the conditions $\alpha_{j}+\beta_{j} \geq 0 \quad \alpha_{j} \beta_{j}>0$ for more careful constants $A_{j}, B_{j} \geq 0$ of highlighting achievements $S_{\alpha_{j}, A_{j}}^{\beta_{j}, B_{j}}$ The current study on a theoretical forward platform Gelfand Shilov type distributional spaces $\left(S_{\alpha_{j}}^{\beta_{j}}\right)^{\prime}\left(\sum_{\alpha_{j}}^{\beta_{j}}\right)$ ' are given by the encouraging smooth intersection union for $A_{j}, B_{j} \geq 0$ of research plan $\left(S_{\alpha_{j}, A_{j}}^{\beta_{j}, B_{j}}\right)^{\prime}$ and its corresponding topological sense is given by promising application of our technique the projective and inductive limits as justifying the acceptability

$\left(S_{\alpha_{j}}^{\beta_{j}}\right)^{\prime}=\bigcap_{A_{2}, B_{2}>0}\left(S_{\alpha_{j}, A_{j}}^{\beta_{j}, B_{j}}\right)^{\prime}$ and $\left(\sum_{\alpha_{j}}^{\beta_{j}}\right)=\bigcup_{A_{2}, B_{2}>0}\left(S_{\alpha_{j}, A_{j}}^{\beta_{j}, B_{j}}\right) '$

Now we are ready to extend construct here the integral representations of the generalized hypergeometric functions to establish new inequalities $\left(S_{\alpha_{j}}^{\beta_{j}}\right)$ ' the dual of $S_{\alpha_{j}}^{\beta_{j}}$ $\left(\sum_{\alpha_{j}}^{\beta_{j}}\right)^{\prime}$ the dual of $\sum_{\alpha_{j}}^{\beta_{j}}$ the systematic theory of straightforward extension of previously thought two dimensional some $S$ type spaces of Laplace Stieltjes transform $S_{\alpha_{i j}, A_{i j}}^{\beta_{i j}, B_{i j}}$ using the expected fact Gelfand Shilov technique for $\alpha_{i j}=\alpha_{i}, \alpha_{j} ; \beta_{i j}=\beta_{i}, \beta_{j}$ influence the results

$$
\begin{aligned}
& \gamma_{a, k, l, q} \varphi=\left\{\varphi \in C^{\infty}\left(R^{d_{i}+d_{j}}\right) / \exists C>0,\right. \\
& \sup _{\substack{0<t<\infty \\
0<x<\infty}}\left|e^{a\left(t_{1},---, t_{m}\right)}(1+x)^{k} D_{\left(t_{1},--, t_{m}\right)}^{l}\left(x D_{x}\right)^{q} \varphi\left(t_{i}, x\right)\right| \\
& \leq C A_{1}^{a}---A_{m}^{a} A^{k} B_{1}^{l}---B_{m}{ }^{l} B^{q} h \\
& \left.a^{a \alpha_{1}}---a^{a \alpha_{m}} k^{k \alpha_{j}} l^{l \beta_{1}}---l^{l \beta_{m}} q^{q \beta_{j}}\right\}
\end{aligned}
$$

we believe our findings compare well with the constants $C ; A_{i j}=A_{i}, A_{j} ; B_{i j}=B_{i}, B_{j} ;(i=1,2,--, m)$

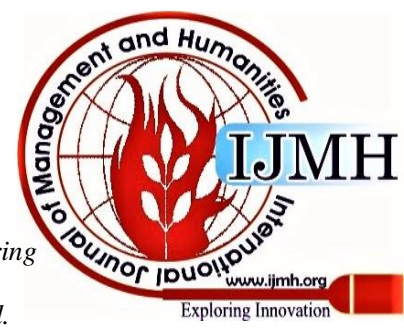




\section{Empirical Analysis of Duality Spaces Connecting Distinct Optical Form Transforms}

depend on the focus of the topic everywhere differential testing function $\varphi$. The possible source spaces $S_{\alpha_{i j}}^{\beta_{i j}, A_{i j}}$ and $\sum_{\alpha_{i j}, A_{i j}}^{\beta_{i j}, B_{i j}}$ the key terminology in field pair of Laplace Stieltjes transform designed for innovative solution act as a very powerful mathematical tool applied in various areas of engineering and science with the increasing complexity of engineering problems are given by the union and intersection for $A_{i j}, B_{i j} \geq 0$ of $S_{\alpha_{i j}}^{\beta_{i j}, B_{i j}}$ and its topology is given by influenced results obtained the inductive and projective limits within the framework of the criteria.

$$
\begin{gathered}
S_{\alpha_{i}, \alpha_{j}}^{\beta_{i}, \beta_{j}}=\text { ind } \lim _{A_{i j}, B_{i j}>0} S_{\alpha_{i j}, A_{i j}}^{\beta_{i j}, B_{i j}} \\
\sum_{\alpha_{i}, \alpha_{j}}^{\beta_{i}, \beta_{j}}=\text { proj } \lim _{A_{i j}, B_{i j}>0} S_{\alpha_{i j}, A_{i j}}^{\beta_{i j}, B_{i j}}
\end{gathered}
$$

We treat the mathematical equipments $S_{\alpha_{i}, \alpha_{j}}^{\beta_{i}, \beta_{j}}$ and $\sum_{\alpha_{i}, \alpha_{j}}^{\beta_{i}, \beta_{j}}$ as nontrivial powerful extension iff there exists more elegant factors $\alpha_{i j}+\beta_{i j} \geq 0$ and $\alpha_{i j} \beta_{i j}>0$ reporting most striking results the union and intersection emerge from the supporting constants $A_{i j}, B_{i j} \geq 0$ of satisfactory confirmation space $S_{\alpha_{i}, A_{i}}^{\beta_{i}, B_{i}}$.

Describing purpose of the Gelfand Shilov method distributional spaces $\left(S_{\alpha_{i}, \alpha_{j}}^{\beta_{i}, \beta_{j}}\right)$ ' and $\left(\sum_{\alpha_{i}, \alpha_{j}}^{\beta_{i}, \beta_{j}}\right)$ ' are given by most well known findings the intersection and union set up used for $A_{i j}, B_{i j} \geq 0$ of $\left(S_{\alpha_{i}, A_{i}}^{\beta_{i}, B_{i}}\right)$ ' and for sake of simplicity its topological sense is straight forwarded on the basis of the projective and inductive limit theory :

$$
\begin{aligned}
& \left(S_{\alpha_{i}, \alpha_{j}}^{\beta_{i}, \beta_{j}}\right)^{\prime}=\bigcap_{A_{i}, B_{i}>0}\left(S_{\alpha_{i j}, A_{i j}}^{\beta_{i j}, B_{i j}}\right)^{\prime} \\
& \left(\sum_{\alpha_{i}, \alpha_{j}}^{\beta_{i}, \beta_{j}}\right)^{\prime}=\bigcup_{A_{i}, B_{i}>0}\left(S_{\alpha_{i j}, A_{i j}}^{\beta_{i j}, B_{i j}}\right)^{\prime}
\end{aligned}
$$

At this point the progressive space is defined by $S_{\alpha_{i}, \alpha_{j}}^{\beta_{i}, \beta_{j},+}=\bigcup_{A_{i}, B_{i}>0} S_{\alpha_{i}, A_{i}}^{\beta_{i}, B_{i},+} \quad$ calculated maximum comparison with the identified topologies and its topology is defined by respective corresponding total families of the inductive limit $S_{\alpha_{i}, \alpha_{j}}^{\beta_{i}, \beta_{j},+}=$ ind $\lim _{A_{i}, B_{i}>0} S_{\alpha_{i}, A_{i}}^{\beta_{i}, B_{i},+}$.

Highlighting limitations of previous sections obviously a well known criticism multiplication by independent variables $t_{1},--, t_{m}, x$ and differentiation there are still uncertainty regarding to continuity in progressive space $S_{\alpha_{i}, \alpha_{j}}^{\beta_{i}, \beta_{j},+}$ a closed subspace $m+1$ kinds of Gelfand Shilov Laplace Stieltjes transform $S_{\alpha_{i}}^{\beta_{i},+}$

or $\alpha_{i}+\beta_{i} \geq 1$ satisfying $m+1$ existing elements in spaces $S_{\alpha_{i}, \alpha_{j}}^{\beta_{i}, \beta_{j},+}$ not only additional localization property sometimes called strip localization but also inspired to control almost exponentially strip localization.
We observe that $\left(S_{\alpha_{i}, \alpha_{j}}^{\beta_{i}, \beta_{j}}\right)^{\prime}$ is the dual of $S_{\alpha_{i}, \alpha_{j}}^{\beta_{i}, \beta_{j}}$ and $\left(\sum_{\alpha_{i}, \alpha_{j}}^{\beta_{i}, \beta_{j}}\right)^{\prime}$ is the dual of $\sum_{\alpha_{i}, \alpha_{j}}^{\beta_{i}, \beta_{j}}$ the ordered topological vector corresponding dual spaces introduced for the study of Cauchy problems in partial differential equations created as a model in technical subjects are the spaces of ultradistributions of Roumieu and Beurling respectively. Unless specified otherwise all the spaces introduced throughout will henceforth be considered equipped with their naturally Hausdorff locally convex pointwise convergent topologies on

these spaces are generated and replaced by the family of bounded convergent topological seminorms $\left\{\gamma_{a, k, l, q}\right\}$.

The considered domain is possible in the present situation $-\infty<t_{i}<0,0<x<\infty$ is in $\hat{S}_{\alpha_{i}, \alpha_{j}}^{\beta_{i}, \beta_{j}}$ without losing any of the original properties if $\varphi$ smooth function $\hat{\varphi}(t, x)=\varphi(-t, x)$ is in $S_{\alpha_{i}, \alpha_{j}}^{\beta_{i}, \beta_{j}}$ the combination transform with some additional features like order properties of the transform satisfying inequality

$$
\begin{aligned}
& \leq C A_{1}{ }^{a}---A_{m}{ }^{a} A^{k} B_{1}^{l}---B_{m}{ }^{l} B^{q} \\
& \quad a^{a \alpha_{1}}---a^{a \alpha_{m}} k^{k \alpha_{j}} l^{l \beta_{1}}---l^{l \beta_{m}} q^{q \beta_{j}}
\end{aligned}
$$

satisfying all above mentioned properties for spaces less than in above sections $\infty$ for which the topology of bounded convergence is assigned to the dual function corresponding to all defined Laplace Stieltjes the spaces in polynomial as well as differential manner having defined domain $-\infty<t_{i}<0,0<x<\infty$

$\hat{S}_{\alpha_{i}}, \hat{S}^{\beta_{i}}, \hat{S}_{\alpha_{j}}, \hat{S}^{\beta_{j}}, \hat{S}_{\alpha_{i}}^{\beta_{i}}, \hat{S}_{\alpha_{j}}^{\beta_{j}}, \hat{S}_{\alpha_{i}, \alpha_{j}}^{\beta_{i}, \beta_{j}}$

We treat various choices of consisting of a number of testing function spaces distributional spaces depending on all defined nondefined used their closed subspaces with analytic signals almost exponentially localized in time and frequency variables control the decay of the transforms independently in each variables if equipped with their naturally Hausdrof locally convex topologies generated by their respective corresponding total families of seminorms are as usal denoted by $T_{\alpha_{i}}, T^{\beta_{i}}, T_{\alpha_{j}}, T^{\beta_{j}}, T_{\alpha_{i}}^{\beta_{i}}, T_{\alpha_{j}}^{\beta_{j}}, T_{\alpha_{i}, \alpha_{j}}^{\beta_{i}, \beta_{j}}$ Moreover all the spaces having domain $-\infty<t_{i}<0,0<x<\infty$ are equipped with their naturally Hausdrof locally convex topologies $\hat{T}_{\alpha_{i}}, \hat{T}^{\beta_{i}}, \hat{T}_{\alpha_{j}}, \hat{T}^{\beta_{j}}, \hat{T}_{\alpha_{i}}^{\beta_{i}}, \hat{T}_{\alpha_{j}}^{\beta_{j}}, \hat{T}_{\alpha_{i}, \alpha_{j}}^{\beta_{i}, \beta_{j}}$.

We extend the space to gain more attention in $S_{\alpha_{i}, \alpha_{j}, p_{i}, p_{j}}^{\beta_{i}, \beta_{j}, n_{i}, n_{j}}$ $S_{\alpha_{i}, \alpha_{j}, p_{i}, p_{j}}^{\beta_{i}, \beta_{j}, n_{i}, n_{j}}=\left\{\varphi \in C^{\infty}\left(R^{d_{i}+d_{j}}\right) / \exists C>0, \mathrm{~d}\right.$

$$
\sup _{\substack{-\infty<t_{i}<\infty \\ 0<x<\infty}}\left|e^{a\left(t_{1},---, t_{m}\right)}(1+x)^{k} D_{\left(t_{1},--, t_{m}\right)}^{l}\left(x D_{x}\right)^{q} \varphi\left(t_{i}, x\right)\right|
$$

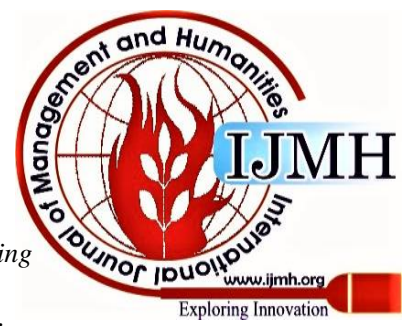




$$
\begin{aligned}
& \leq C\left(A_{1}+\delta_{1}\right)^{a}---\left(A_{m}+\delta_{m}\right)^{a}\left(A_{j}+\delta_{j}\right)^{k} \\
& \left(B_{1}+\eta_{1}\right)^{l}---\left(B_{m}+\eta_{m}\right)^{l}\left(B_{j}+\eta_{j}\right)^{q} \\
& \left.a^{a \alpha_{1}}---a^{a \alpha_{m}} k^{k \alpha_{j}} l^{l \beta_{1}}---l^{l \beta_{m}} q^{q \beta_{j}}\right\} \\
& \quad \text { for } i=1,--, m \delta_{i}, \delta_{j}, \eta_{i}, \eta_{j} \text { are any } m \text { elements }
\end{aligned}
$$

in Laplace sense at the same time one from Stieltjes scene greater than zero whose graph does not have any type of breaks or holes lose the property of strongly continuity at $x=0, t_{i}=0$ hence $m+1$ all variables being strongly continuous at $0<x<\infty,-\infty<t_{i}<\infty$ equipped with their naturally Hausdrof locally convex topologies for each $m+1$ elements including for $i=1,---, m$ generated by their respective corresponding total families of seminorms as usual denoted by $T_{\alpha_{i}, \alpha_{j}, B_{i}, B_{j}}^{\beta_{i}, \beta_{j}, A_{i}, A_{j}}$ for the notable domain

$$
0<x<\infty, 0<t_{i}<\infty \text { and } \hat{S}_{\alpha_{i}, \alpha_{j}, B_{i}, B_{j}}^{\beta_{j}, A_{j}, A_{j}} \text { for the }
$$

domain $-\infty<t_{i}<0, \quad 0<x<\infty$ possess several nuclear invariant convenient mapping properties as a new proposed route demands an advanced imaging technique to resolve the magnetism of nanostructure_under translation dilation by a positive factor in $m+1$ dimension.

\section{CONCLUSION}

The interesting approach from the special study points on the development of the necessary facts related to the generalized functions theory by Schwartz hence the construction of Laplace Stieltjes transform towards reduction the complexity of engineering problems extensively contribution from a topological point of aspect in_along with the decrease of the computation time of simulations these techniques have significantly contributed to magnetic phenomenon thereby boosting the novel technological applications in the respective field by introducing convenient explanations for more general situations to achieve enjoy a slightly faster decay in domain even in polynomial case by changing the scheme from one dimension to higher by successfully employing the relative testing function space.

\section{REFERENCES}

1. A. H. Zemanian, Realizability Theory for Continuous Linear Systems Academic Press, New York.1968.

2. Yu A. Brychkov, A. P. Prudnikov, Integral transforms of Generalized Functions. Gordon and breach science publishers. 1968.

3. María Ángeles García-Ferrero, Angkana Rüland, "Strong unique continuation for the higher order fractional Laplacian," Mathematics in Engineering. 2019, 1(4), pp. 715-774.

4. P. A. Gulhane \& A. S. Gudadhe, "On L.S. Spaces of Gelfand Shilov Technique," Bulletin of Pure \& Applied Sciences, Vol 25E, No. 1, 2006, pp. 351- 354.

5. K. Grochenig, G. Zimmermann, "Spaces of test functions via the STFT," Journal of Function Spaces and Applications. 2004, 2(1), pp. 25-53.

6. D. Andreas, J. Javier, S. Javier, "Injectivity \& Surjectivity of the Stieltjes Moment mapping in Gelfand-Shilov spaces," arXiv:1902.01305vI [Math.FA] 4Feb2019.

7. MC Garne, "A generalized Laplace Stieltjes transformation," Proceeding of the American Mathematical Society, Vol 8, No. 2, 1957, pp. 278-285.

8. YY Kong, D. C. Sun, "On the growth properties of the Laplace Stieltjes transform," Complex Var Elliptic Eqn, 59, 2014, pp.553-563.
9. YY Kong, Y. Yang, "On Type function \& the growth of Laplace Stieltjes transformations convergent in the right half plane," Adv Math, 37(2), 2007, pp. 197-205.

10. YY Kong, YY Hu, “On generalized orders \& types of Laplace Stieltjes transforms analytic in the right half plane," Acta Math Sin 59, 2016, pp 91-98.

11. YY Kong, X. Luo, "On the order \& type of Laplace Stieltjes transforms of slow growth," Acta Math Sin, 55(1), 2012, pp. 141-148.

12. C. Singhal, G. S. Srivastava, "On the growth \& approximation of entire functions represented by Laplace Stieltjes transformation," Ann Uni Ferrara doi:10.1007/s11565017-0272,2017.

13. L. Deshna, "Solutionof Integral Equations \& Laplace Stieltjes transforms," Palestine Journal of Mathematics, Vol 5(1) , 2016, pp 43-49.

14. Hong Yan $\mathrm{Xu}$, "The approximation of Laplace Stieltjes transforms with finite order," Journal Inequal Appl, 2017(1), 164, doi:10.1186/s13660-017-1441-9,xhyhhh126.com.

15. P. Kree, "La Theorie des Distributions en Dimension Quelconque et Integration Stochastique," Lecture Notes in Math,136, Springer-Verlag, 1988, pp. 170-233.

\section{AUTHORS PROFILE}

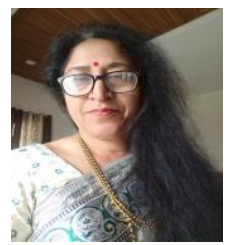

Padmaja G, PhD Mathematics in year 2008, PG diploma in Computer Science, Assistant Professor a Govt College of Engg Amravati, 33 Journal Publications on research work including Scopus, UGC approved, high impact factor, accepted, communicated.

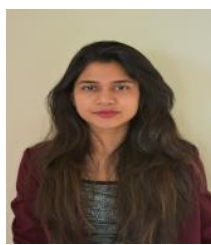

Abhilasha Gulhane , Research Assistant at UIUC

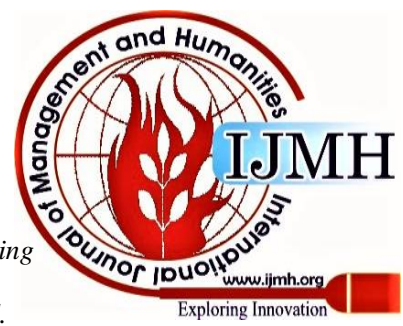

